\title{
Design of Self-restriction Hydrostatic Thrust Spherical Bearing (Fitted Type)
}

\author{
Ahmad Waguih Yacout Elescandarany \\ Mechanical Department, Faculty of Engineering, Alexandria University, Alexandria, Egypt \\ Email address: \\ awaguih@yahoo.com
}

To cite this article:

Ahmad Waguih Yacout Elescandarany. Design of Self-restriction Hydrostatic Thrust Spherical Bearing (Fitted Type). International Journal of Mechanical Engineering and Applications. Vol. 7, No. 4, 2019, pp. 111-122. doi: 10.11648/j.ijmea.20190704.14

Received: August 14, 2019; Accepted: September 4, 2019; Published: September 20, 2019

\begin{abstract}
This is the last part of the series studying the fitted hydrostatic thrust spherical bearing. It handles an unconventional design of this type of bearings. The conception of this design is to break the rules controlling the bearing restrictions, where it is believed that without restrictors no hydrostatic bearing could be got (axiom). The paper focused the effort to derive a general characteristic equation that can control the design in turn the bearing performance and behavior. This general characteristic equation, through its simple form, gives the designer the ability to get a comprehensive conception about his problem and widely opens the door in front of him to design a conventional or unconventional bearing whatever the bearing purpose. The effective parameters; needed to be known for designing the bearing; were concentrated into three items; the rotor speed, the seat dimensions and the lubricant properties. The characteristic equation shows that the seat radius and the inlet angle play the major role in determining the supply pressure, in turn the load carrying capacity. The inertia, the recess angle and the lubricant viscosity have the major effect on determining the bearing stiffness in case of the partial hemispherical seats while in case of the hemispherical seats the stiffness has slightly been affected. The design shows that the bearings with hemispherical seats have extremely low stiffness, practically zero stiffness and very high temperature rise, which make this bearing configuration invalid to be self restriction bearing; while the bearings with partial hemispherical seats have a very wide stiffness range allowing the designer to control and design the bearing with the stiffness needed for any purpose (from zero stiffness to extremely high stiffness). The lubricant temperature rises about three degrees centigrade which practically means that the bearing operates at constant temperature.
\end{abstract}

Keywords: Hydrostatic Bearings, Spherical Bearings Design, Surface Roughness, Inertia, Viscosity Effects, Self Compensation

\section{Introduction}

The previous researches offered plenty of the traditional studies and designs of the hydrostatic thrust spherical bearing with restrictors or with self-compensation.

Ahmad W. Yacout [1-4] studied the fitted type, with and without recess, of the hydrostatic thrust spherical bearing with capillaries and orifices restrictors finding the effects of the inertia, surface roughness and lubricant fluid viscosity on the beating performance and offering an optimal design of this bearing.

Kane N. R. et al [5] offered a design of a hydrostatic rotary bearing with angled surface self-compensation consisting of five precisely machined parts provided with a sealing system. It is concluded that the novel hydrostatic bearing is potentially useful for applications that require very high rotational precision and stiffness in a low profile package.

Xiaobo Z. et al [6] investigated the influence of design parameters on the static performance of a designed selfcompensating hydrostatic rotary bearing where the results showed that the optimum designed resistance ratio is 1 ; the initial clearance ratio should be small, and the inner resistance ratio should be large.

Yuan K. et al [7] studied a hydrostatic bearing with doubleaction variable compensation of membrane-type restrictors (DAMR) and self-compensation (SC) where it is concluded that the critical (ddc) dimensionless deformation coefficient value of DAMR which corresponds to infinite stiffness is $(2 / 3)$ and the static characteristics of self-compensation (SC) are always same as that of constant restrictors. 
Xu C. and Jiang S. [8] conducted a comparative study of the static behavior between the self-compensation hydrostatic spherical hinge and the hydrostatic spherical hinge with orifice restrictor where the results showed that the selfcompensation hydrostatic spherical hinge has an advantage in the static behavior over the hydrostatic spherical hinge with orifice restrictor, including a much larger load capacity, a smaller flow rate, and a smaller power loss.

Khaksea P. G. et al [9] offered a comparative study for the performance of a non-recessed hole-entry Hybrid/ Hydrostatic conical bearing compensated with capillary and orifice Restrictors where it is concluded that, in general, orifice compensated non-recess conical journal bearing showed higher performance characteristics as compared to the counterpart bearing for the applied radial load.

Zhifeng $\mathrm{Li}$ et al [10] offered a fruitful review of hydrostatic bearing system where the articles about hydrostatic bearings since 1990 were collected in this review. Researching status was evaluated in two aspects: basic theory and typical application. Basic theory contains equations and analysis methods which include analytic, numerical, and experimental methods. Typical applications were based on rectangular oil pad, circular oil pad, and journal bearings. Moreover, the review focused on the analysis of the relevant model, solution, and optimization and summarized the hotspots and development directions.

Alexander S. [11-12] discussed and compared between bearings with restrictors and others of self compensation using different lubricants (oil \& water) where the study proved the priority of the self compensation type numerating its advantages and concluding that it makes the system far less sensitive to contamination especially if water is used, it provides greater stiffness and load capacity and it makes the system insensitive to manufacturing tolerances. Besides the bearings are self-tuning where the stiffness automatically optimizes itself for the bearing as soon as it is turned on (no manual tuning of capillary or orifice size is required) and the bearing is not significantly affected by large gap variations caused by manufacturing tolerances which make the bearing suitable for use with water or water based coolant as a bearing lubricant.

Z. Y. Dong et al [13] obtained the relationships of worktable displacement, load capacity, and static stiffness by using flow continuity equations of a self-compensated hydrostatic bearing. The results revealed that the appropriate range of design parameters for self-compensated hydrostatic bearing can obtain the maximum stiffness.

Mohit Agarwal [14] studied the stiffness of an opposed pad hydrostatic bearing showing that the performance of a hydrostatic bearing can be improved over a certain range of load capacity if the design parameters of the restrictor set-up are properly chosen where the design restriction ratio should be chosen differently for lower and higher loading conditions for high static stiffness.

Antony Wong [15] presented, through the master thesis, a design and manufacturing method for a new surface self compensating hydrostatic bearing where a lumped resistance model was used to analyze the performance of the bearing and provide guidance on laying out the bearing features; concluding the results of the model indicate the design is extremely robust.

The present design of hydrostatic thrust spherical bearing is utilizing a new untraditional technique, self-restriction, which helps the designer to get rid of the compensators allowing very low design complexity.

\section{Mathematics}

The mathematical bearing expressions are listed in the appendix.

\subsection{Bearing General Characteristic Equation}

\subsubsection{Bearing with Orifice Restrictor}

From Yacout [1-4]

$$
\begin{aligned}
& q=K_{o}\left[\frac{p_{s}}{3}\right]^{\frac{1}{2}} \\
& K_{o}=\frac{\pi C_{d} d_{o}^{2}}{\sqrt{8 \rho}} \\
& C_{d}=0.6 \rightarrow \operatorname{Re}_{o}>15 \\
& d_{o}>5 \times 10^{-4} m \\
& m_{o}=\frac{d_{o}}{d_{o i}} \\
& Q=\frac{9 \mu q}{\pi e^{3} p_{s}} \\
& \operatorname{Re}_{o}=\frac{d_{o}}{\mu}\left[\frac{2 \rho p_{s}}{3}\right]^{\frac{1}{2}}
\end{aligned}
$$

From 1, 2:

$$
e^{6} Q^{2}=\left(1.215 m_{o}^{4}\right)\left(\frac{\mu^{2}}{\rho p_{s}}\right) d_{i o}^{4}
$$

From 3, 4:

$$
e^{3} Q=\left(\frac{0.9}{\operatorname{Re}_{o}}\right) m_{o}^{3} d_{i o}^{3}
$$

\subsubsection{Bearing with Capillary Tube Restrictor}

$$
\begin{aligned}
& q=\left(\frac{K_{c a p}}{3 \mu}\right) p_{s} \\
& K_{c a p}=\frac{\pi d_{c}^{4}}{128 l_{c}}, \\
& n_{c}=\frac{l_{c}}{d_{i o}} \geq 20 \\
& m_{c}=\frac{f_{c}}{d_{i o}} \\
& \operatorname{Re}_{c} \leq 2000
\end{aligned}
$$


From equations $(2,6)$ :

$$
e^{3} Q=\left(\frac{3}{2560}\right) m_{c}^{3} d_{i o}^{3}
$$

\subsubsection{The Relation Between the Two Restrictors}

From equating equations $(5,7)$ :

$$
\operatorname{Re}_{o}=768 \quad \& \quad \operatorname{Re}_{c}=2000
$$

Hence, the general characteristic governing equation for this type of bearings is:

$$
\begin{aligned}
& e^{3} Q=\left(\frac{0.9}{768}\right) m_{o}^{3} d_{i o}^{3} \quad \text { or } \\
& e^{3} Q=\left(\frac{3}{2560}\right) m_{c}^{3} d_{i o}^{3}
\end{aligned}
$$

Put:

$$
\begin{gathered}
m_{c}=m_{o}=m \\
e^{3} Q=\left(1.172 \times 10^{-3}\right) m^{3} d_{i o}^{3}
\end{gathered}
$$

Equation (9) is the general characteristic governing equation for this type of bearings.

\subsection{Restrictor-less Bearing}

Equating $(m)$ with the unity, equation ( 8 or 9 ) becomes:

$$
\begin{aligned}
e^{3} Q & =\left(\frac{3}{2560}\right) d_{i o}^{3} \text { or } \\
e^{3} Q & =\left(1.172 \times 10^{-3}\right) d_{i o}^{3}
\end{aligned}
$$

\section{Restrictor-less Bearing Design}

Generally, Equation (9) could be used to design a bearing with or without restrictors as needed and equation (10) could directly be used for designing the restrictor-less (self restriction) bearing.

\subsection{Determining the Supply Pressure}

From equations $(4,9)$ :

$$
\begin{gathered}
\left(\frac{3}{2560}\right)^{2} d_{i o}^{6}=(1.215 \times 1)\left(\frac{\mu^{2}}{\rho p_{s}}\right) d_{i o}^{4} \\
p_{s}=\left(0.884736 \times 10^{6}\right)\left(\frac{\mu^{2}}{\rho d_{i o}^{2}}\right) \\
d_{i o}=2 R \sin \phi_{i} \\
p_{s}=\left(0.221184 \times 10^{6}\right)\left(\frac{\mu^{2}}{\rho R^{2} \sin ^{2} \phi_{i}}\right)
\end{gathered}
$$

\subsection{Determining the Seat Inlet Angle ( $\left.\phi_{i}\right)$}

The angle $\left(\phi_{i}\right)$ could be determined as:

$$
\begin{aligned}
& S=\frac{9 \rho \Omega^{2} R^{2}}{80 P_{s}} \\
& P_{s}=\frac{9 \rho \Omega^{2} R^{2}}{80} \\
& \Omega=2 \pi N
\end{aligned}
$$

Relating with equation (11), then:

$$
\begin{aligned}
& \sin \phi_{i}=\left(\frac{700 \mu \sqrt{S}}{\rho N a}\right) \\
& \phi_{i}=\sin ^{-1}\left(\frac{700 \mu \sqrt{S}}{\rho N a}\right)
\end{aligned}
$$

To get the best benefit from the inertia in the design, the speed parameter $(\mathrm{S}=1)$

\subsection{Determining the Design Eccentricity}

From equations $(13,15$ and 16$)$ the quantity $\left(e^{3} Q\right)$ could be calculated at different values of (e) at the design speed parameter $(S)$. Drawing this quantity against (e), the design eccentricity (e) which satisfies equation (10) could be graphically determined in turn the design $(Q)$.

\subsection{Determining the Load Carrying Capacity}

Getting the design (e), the inlet and exit bearing angles $\left(\theta_{i} \& \theta_{e}\right)$ could be calculated as in Yacout [1-4], the dimensionless load carrying capacity $(W)$ could be calculated from equation (17).

\subsection{Determining the Total Losses $\left(P_{t}\right)$}

From equations (18-21) the total bearing losses could be calculated.

\subsection{Temperature Rise and Distribution}

From equations (22-23) the temperature rise could be theoretically and numerically calculated.

\subsection{Determining the Bearing Stiffness}

The theoretical or numerical stiffness calculations of this type of bearings are extremely tedious due to the crescent shape of the film thickness and the complicated relation between the load and the film thickness. However, the stiffness will be handled in some details based on the film thickness direction.

\subsubsection{Axial Bearing Stiffness $\left(\lambda_{a}\right)$}

Putting into consideration that the film thickness in the thrust direction is:

$$
h_{a}=e \cos ^{2} \theta
$$

The stiffness could be calculated numerically in the thrust direction following the same procedures as found in the appendix to be:

$$
\lambda_{a}=\sum_{l=1}^{l}\left(\lambda_{a}\right)_{l}
$$




\subsubsection{Transversal Bearing Stiffness $\left(\lambda_{t}\right)$}

The film thickness in the transversal direction is:

$$
h_{t}=e \cos \theta \sin \theta
$$

Hence:

$$
\lambda_{t}=\sum_{l=1}^{l}\left(\lambda_{t}\right)_{l}
$$

\subsubsection{Radial Bearing Stiffness $\left(\lambda_{r}\right)$}

The film thickness in the transversal direction is:

$$
h_{r}=e \cos \theta
$$

Hence:

$$
\lambda_{r}=\sum_{l=1}^{l}\left(\lambda_{r}\right)_{l}
$$

\subsubsection{Internal Bearing Stiffness $\left(\lambda_{\theta}\right)$}

The detailed theoretical derivation is in the appendix where:

The internal localized stiffness is:

$$
(\lambda)_{\theta}=\left(\frac{2 \pi R^{2} p}{e}\right) \cos \theta
$$

And the mean internal stiffness is:

$$
\lambda_{\theta}=\sum_{\theta=\theta_{i}}^{\theta_{e}}(\lambda){ }_{\theta}
$$

\section{Design Example}

It is required to design a high speed bearing with $(50 \mathrm{~mm})$ radius, maximum rotor speed $(N=500 r . p . s)$ and intended $\operatorname{load}(L=20 \mathrm{KN})$.

\section{Design Procedures}

\subsection{Selections}

\subsubsection{Lubricant Fluid Properties}

$\left(\mu=0.086\right.$ N.s $/ \mathrm{m}^{2}, \mathrm{~K}_{\mathrm{v}}=0.5, \rho=867 \mathrm{~N} . \mathrm{s}^{2} / \mathrm{m}^{4}, \mathrm{C}_{\mathrm{v}}=1880$ $\left.\mathrm{J} / \mathrm{Kg} \cdot \mathrm{c}^{0}\right)$

\subsubsection{Geometrical Dimensions}

$\phi_{e}=\eta \pi / 2, \phi_{i}($ to be calculated $) \phi_{r}=Y \phi_{e}, \eta=0.81, Y=0.3$

\subsubsection{Surface Roughness Specifications}

$$
(\xi=0.05)
$$

\subsection{Design Calculations}

\subsubsection{Determining the Seat Inlet Angle ( $\left.\phi_{i}\right)$}

From equation (12):

$$
\begin{aligned}
& \sin \phi_{i}=\left(700 \times 0.086 / 867 \times 500 \times \pi \times 0.05^{2}\right)=0.014 \\
& \phi_{i}=\sin ^{-1}(0.014) \\
& \phi_{i}=0.8^{\circ} \\
& d_{i o}=2 R \sin \phi_{i} \\
& d_{i o}=1.4 \quad \mathrm{~mm}
\end{aligned}
$$

\subsubsection{Determining the Supply Pressure ( ${ }^{P_{s}}$ )}

From equation (11):

$$
\begin{aligned}
& P_{s}=\left(0.221184 \times 10^{6}\right)\left(\frac{\mu^{2}}{\rho R^{2} \sin ^{2} \phi_{i}}\right) \\
& P_{S}=\left(\frac{0.221184 \times 10^{6} \times 0.068^{2}}{867 \times 0.05^{2} \times 0.014^{2}}\right) \\
& P_{s}=2.41 \times 10^{6} \mathrm{~N} / \mathrm{m}^{2}
\end{aligned}
$$

\subsubsection{Determining the Eccentricity (e)}

The quantity $\left(e^{3} Q\right)$ could be easily calculated at different values of (e), drawn against (e) and the design eccentricity which satisfies equation (10) could be graphically determined in turn the design $(\mathrm{Q})$.

From figure 1-a:

$$
\begin{aligned}
& \mathrm{e}^{3} Q=3.2023 \times 10^{-12} \\
& e=168.2 \mu \mathrm{m} \\
& Q=0.6725
\end{aligned}
$$
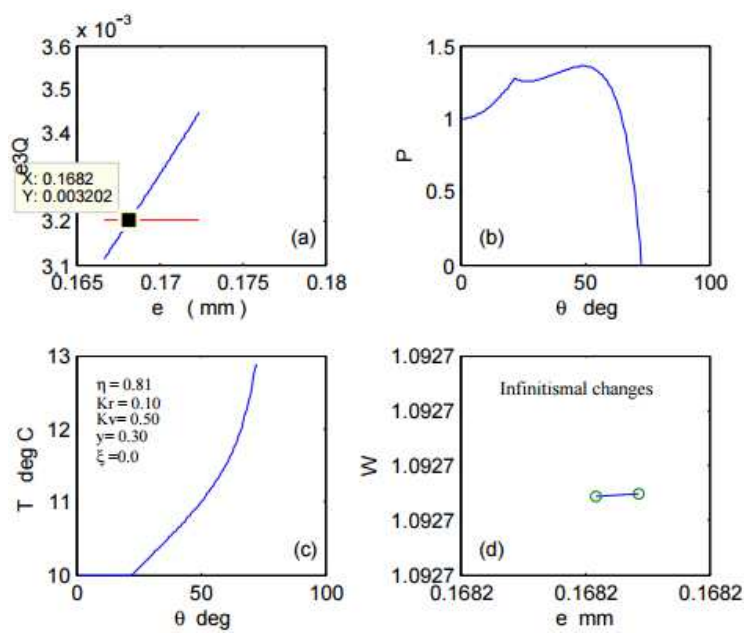

Figure 1. Partial with recess.

\subsubsection{Determining the Load Capacity (w)}

From equation (17) the load could be calculated to be:

$$
w=20.718 K N
$$

Hence, the load factor of safety $\left(f_{s}\right)$ is:

$$
\begin{aligned}
& f_{s}=\frac{w}{L}=\frac{20.718 \times 10^{3}}{20 \times 10^{34}} \\
& f_{s}=1.0359
\end{aligned}
$$


So, this load should not be exceeded.

\subsubsection{Determining the Flow Rate (q)}

$$
\begin{aligned}
& q=\frac{\pi\left(e^{3} Q\right) \beta p_{s}}{6 \mu} \\
& q=\frac{\pi \times\left(3 . x 120230^{-12}\right) \times(2 / 3) \times\left(2.4141 \times 10^{6}\right)}{6 \times 0.068} \mathrm{~m}^{3} / \mathrm{s} \\
& q=3.9684 \times 10^{-5} \mathrm{~m}^{3} / \mathrm{s} \\
& q=3.9684 \times 10^{-5}\left(3600 \times 10^{3}\right) \quad \mathrm{l} / \mathrm{hr} \\
& q=142.864 \mathrm{l} / \mathrm{hr}
\end{aligned}
$$

5.2.6. Determining the Pump Power $\left({ }^{P_{p}}\right)$

From equation (19):

$$
\begin{aligned}
& p_{p}=q \cdot p_{s} \\
& P_{p}=\left(3.9684 \times 10^{-5}\right) x\left(2.41 \times 10^{6}\right) \\
& P_{p}=95.77 \quad \text { N.m/s }
\end{aligned}
$$

\subsubsection{Determining the Temperature Rise ( $\Delta T)$}

The temperature rise could be theoretically calculated as:

$$
\begin{aligned}
\Delta T & =\frac{P_{p}}{\rho q C_{v}} \\
\Delta T & =\frac{95.77}{867 \times 1880 \times 3.9684 \times\left(10^{-5}\right)} \\
\Delta T & =1.48 C^{o}
\end{aligned}
$$

Or numerically by equation (22) and from the temperature distribution figure 1:

$$
\Delta T=2.86 C^{o}
$$

\subsubsection{Determining the Stiffness}

Applying equations (24-29) the localized stiffness and the mean stiffness could be determined.

a) Determining the radial Stiffness $\left(\lambda_{R}\right)$

From figure 2- $a$ \& table 1

$$
\lambda_{R}=110 \quad N / \mu m
$$

b) Determining the axial stiffness $\left(\lambda_{A}\right)$

From figure $2-b \&$ table 1

$$
\lambda_{A}=188.4 \quad N / \mu m
$$

c) Determining the transversal stiffness $\left(\lambda_{T}\right)$

From figure 2-c \& table 1

$$
\lambda_{T}=347 \quad N / \mu m
$$

d) Determining the internal stiffness $\left(\lambda_{\theta}\right)$

$$
\lambda_{\theta}=132 \quad N / \mu m
$$
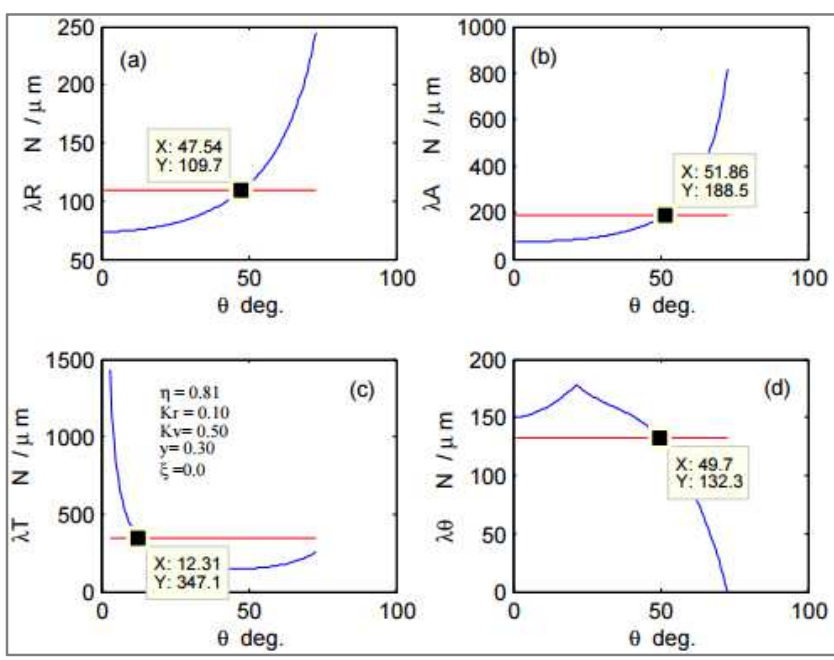

Figure 2. Partial with recess.
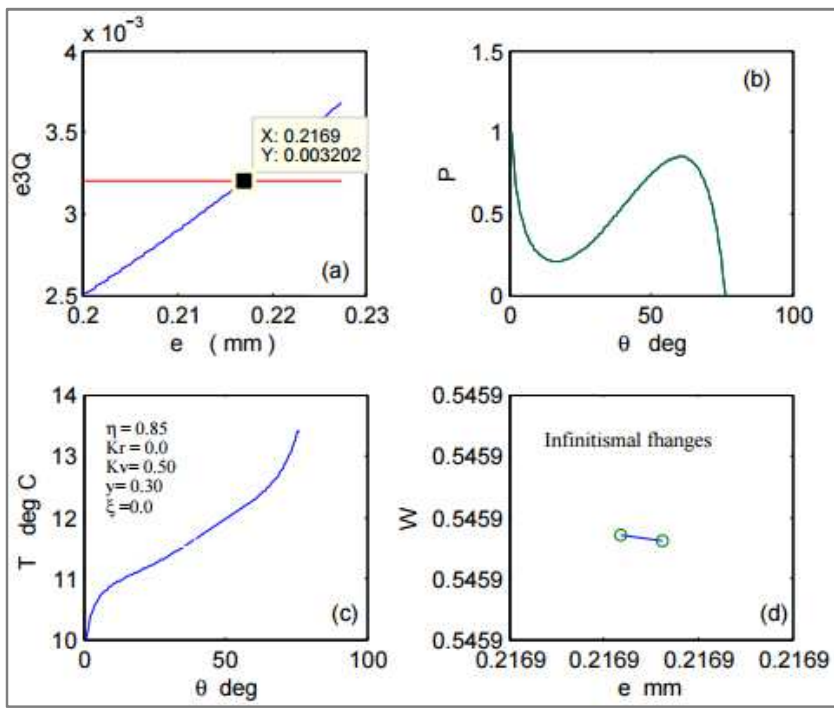

Figure 3. Partial without recess.
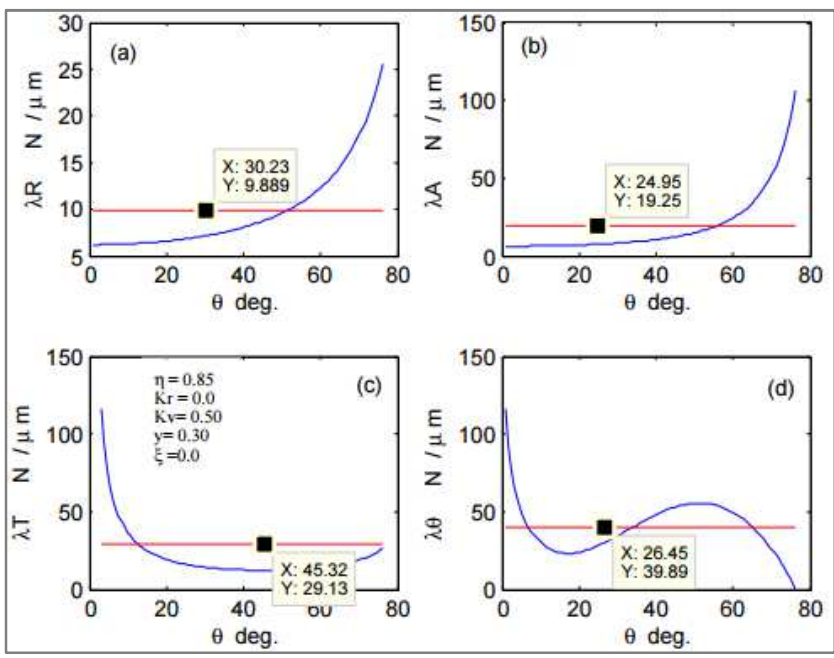

Figure 4. Partial without recess.

From figure 2- $d \&$ table 1 

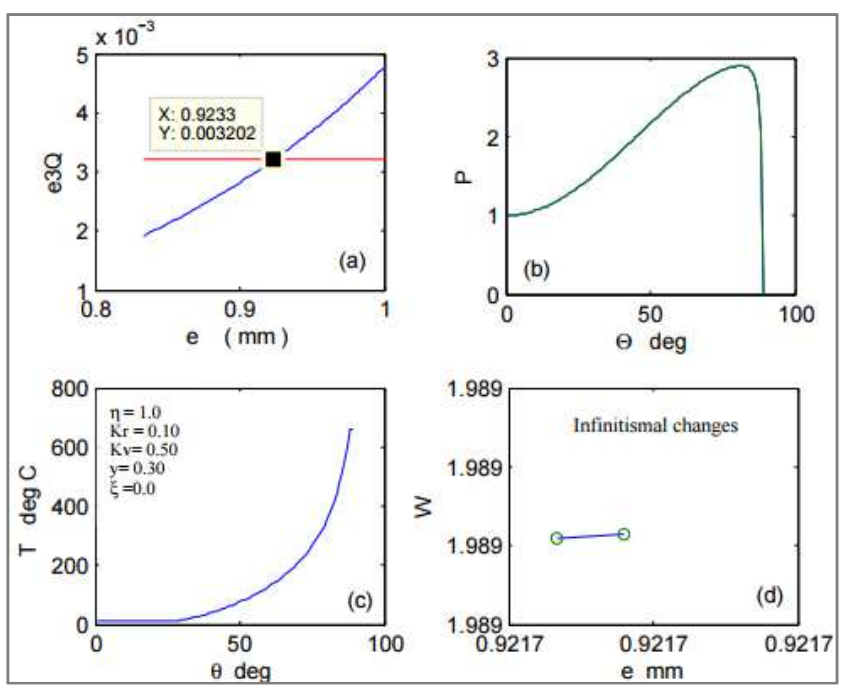

Figure 5. Hemi with recess.
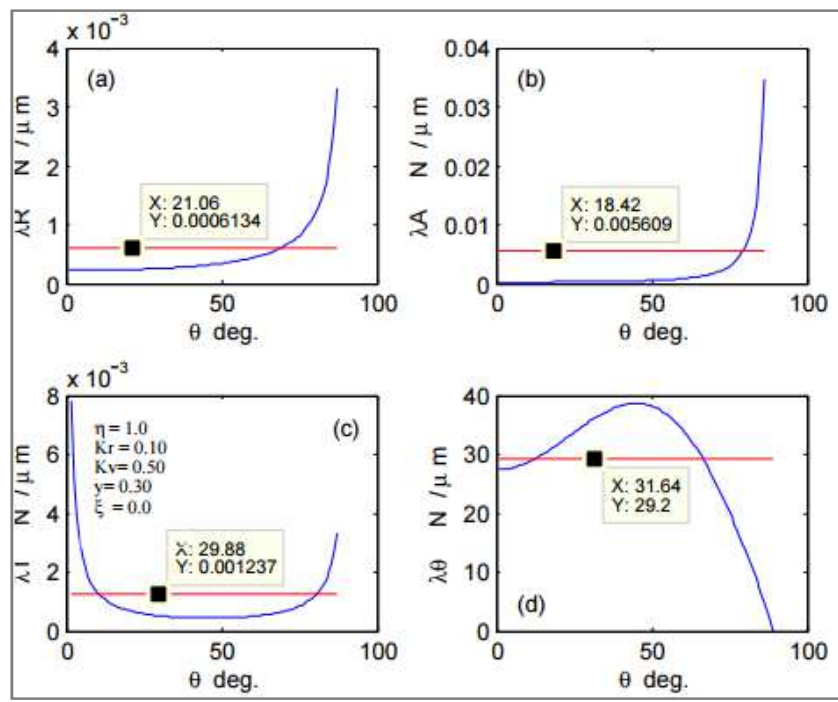

Figure 6. Hemi with recess.
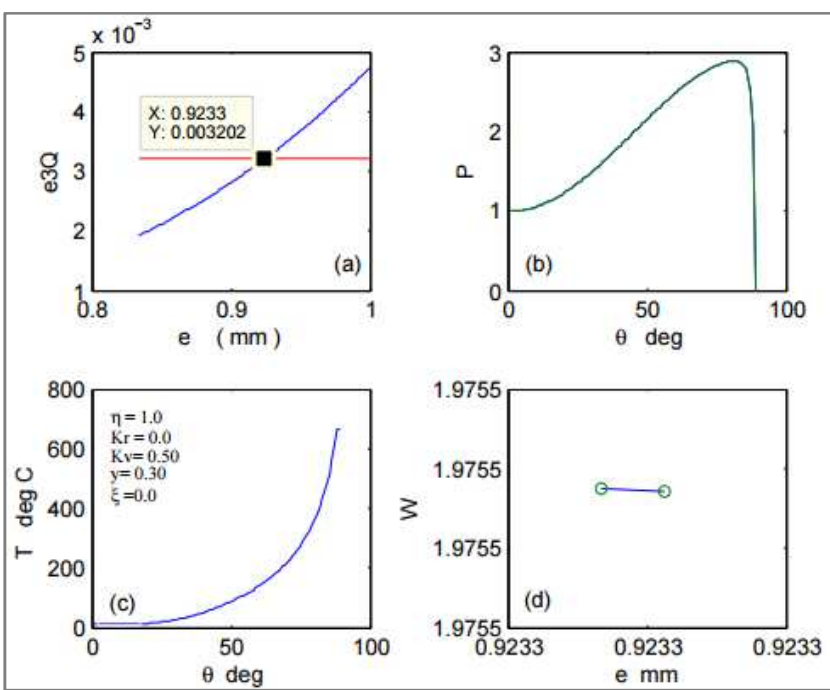
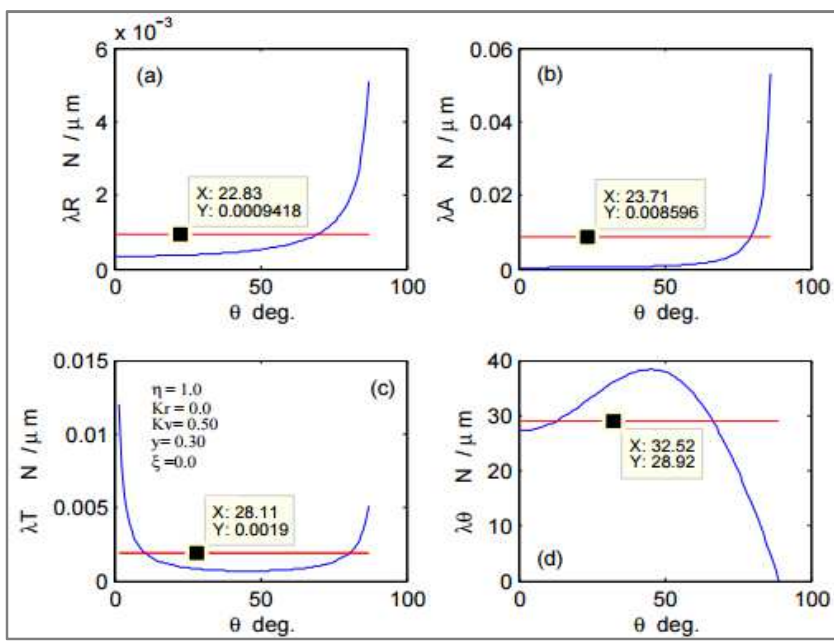

Figure 8. Hemi without recess.
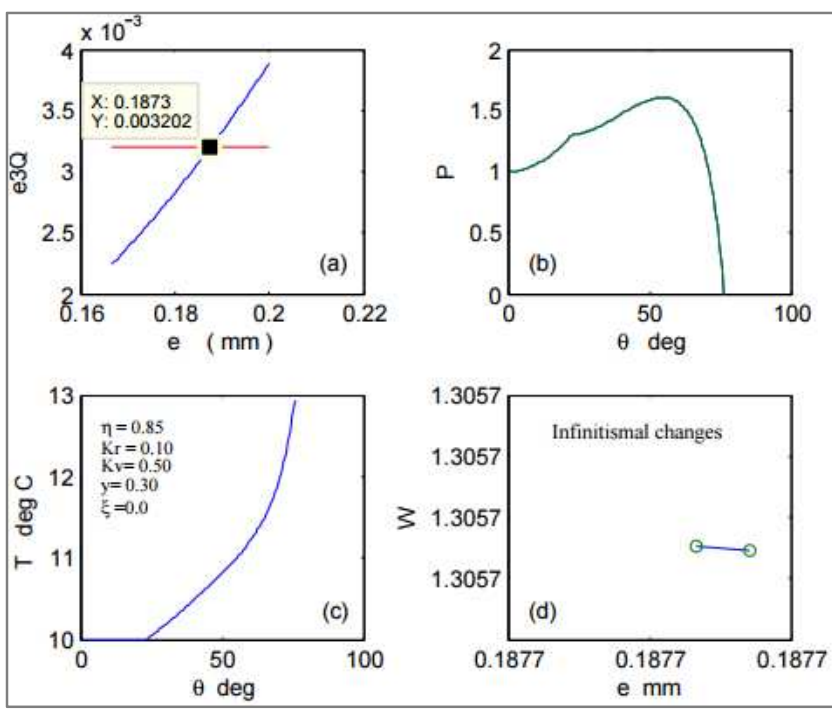

Figure 9. Partial hemi with recess for comparison.
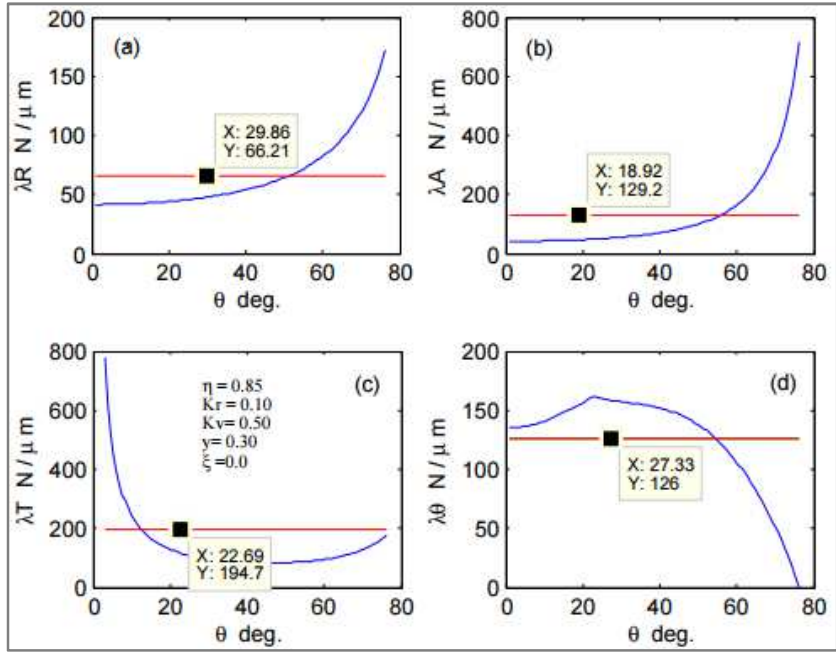

Figure 10. Partial hemi with recess for comparison.

Figure 7. Hemi without recess. 
Table 1. Collecting the bearing data.

\begin{tabular}{|c|c|c|c|c|}
\hline \multirow{2}{*}{$\begin{array}{l}\text { Bearing } \\
\text { Parameters }\end{array}$} & \multicolumn{2}{|c|}{ Without recess } & \multicolumn{2}{|c|}{ With recess } \\
\hline & H.S & P.H. S & H. S & P. H. S \\
\hline$\xi$ & 0.05 & 0.05 & 0.05 & 0.05 \\
\hline$\eta$ & 1 & 0.81 & 1 & 0.81 \\
\hline$\varphi \mathrm{i}(\mathrm{deg})$ & 0.8 & 0.8 & 0.8 & 0.8 \\
\hline$\varphi r \approx$ & 0 & 0 & 27 & 21.9 \\
\hline$\varphi \mathrm{e} \approx$ & 90 & 72.9 & 90 & 72.9 \\
\hline$\Delta(\mathrm{mm})$ & 0 & 0 & 5 & 5 \\
\hline dio $\approx$ & 1.4 & 1.4 & 1.4 & 1.4 \\
\hline $\mathrm{R} \approx$ & 50 & 50 & 50 & 50 \\
\hline Aw $(\mathrm{cm}) 2$ & 79 & 79 & 79 & 79 \\
\hline Load (KN) & 20 & 20 & 20 & 20 \\
\hline $\mathrm{N}$ (r. p. s) & 500 & 500 & 500 & 500 \\
\hline$\rho(\mathrm{N} . \mathrm{s} 2 / \mathrm{m} 4)$ & 867 & 867 & 867 & 867 \\
\hline$\mu(\mathrm{N} . \mathrm{s} / \mathrm{m} 2)$ & 0.068 & 0.068 & 0.068 & 0.068 \\
\hline $\mathrm{Cv}$ (J/ Kg.c) & 1880 & 1880 & 1880 & 1880 \\
\hline$\theta \mathrm{i}$ (degree) & 0.79 & 0.8 & 0.79 & 0.8 \\
\hline$\theta \mathrm{r} \approx$ & 0 & 0 & 26.5 & 21.8 \\
\hline$\theta \mathrm{e} \approx$ & 89 & 72.7 & 89 & 72.7 \\
\hline$\beta$ (dim-less) & $2 / 3$ & $2 / 3$ & $2 / 3$ & $2 / 3$ \\
\hline Ps (MN / m2) & 2.41 & 2.41 & 2.41 & 2.41 \\
\hline e $(\mathrm{mm})$ & 0.9233 & 0.203 & 0.9217 & 0.1682 \\
\hline he $(\mu \mathrm{m})$ & 17 & 60.5 & 17 & 50 \\
\hline Ke (dim-less) & $1 / 54.2$ & $1 / 246$ & $1 / 60$ & $1 / 300$ \\
\hline$S \approx$ & 1 & 1 & 1 & 1 \\
\hline $\mathrm{w}(\mathrm{KN})$ & 38.45 & 5.29 & 38.7 & 20.718 \\
\hline fs $=(w / L)$ & 1.87 & 0.264 & 1.88 & 1.0359 \\
\hline Q (dim-less) & 0.0041 & 0.3802 & 0.0041 & 0.6725 \\
\hline $\mathrm{q}(\mathrm{m} / \mathrm{hr})$ & 0.1429 & 0.1429 & 0.1429 & 0.1429 \\
\hline $\mathrm{Pp}(\mathrm{N} . \mathrm{m} / \mathrm{s})$ & 95.43 & 95.62 & 95 & 95.77 \\
\hline$\lambda \mathrm{A}(\mathrm{N} / \mu \mathrm{m})$ & 0.009 & 12.8 & 0.0056 & 188.4 \\
\hline$\lambda \mathrm{R} \approx$ & 0.0009 & 8.5 & 0.0006 & 110 \\
\hline$\lambda \mathrm{T} \approx$ & 0.002 & 23.7 & 0.0012 & 347 \\
\hline$\lambda \theta \approx$ & 29 & 25 & 29.2 & 132 \\
\hline$\Delta \operatorname{Tn}($ deg. c) & 656 & 3.33 & 649 & 2.86 \\
\hline$\Delta$ Tth (deg. c) & 1.48 & 1.48 & 1.47 & 1.48 \\
\hline
\end{tabular}

\section{Bearing Configuration Checking}

The selected configuration is checked to find out the effect of different parameters $\left(\eta, \theta_{r}, k_{v}, \xi\right)$ on the design.

\subsection{Effect of $(\eta)$}

The seat arc length is lengthened to be (0.85) of the hemispherical one to be put in comparison with the designed one.

\subsection{Effect of (y)}

The recess arc length is extended to be (0.4) of seat arc one through increasing recess angle $\left(\theta_{r}\right)$.

\subsection{Effect of $\left(k_{v}\right)$}

The lubricant is treated as a constant fluid viscosity i.e. $\left(k_{v}=0\right)$.

\subsection{Effect of $(\xi)$}

The bearing surface is treated as an utmost allowed roughened conformal surface in the hydrodynamic regime i.e. $(\xi=1)$.

\section{Results}

As previously stated, it is the last part of a study handling this type of bearings. A new technique is used to design an unconventional bearing. A general characteristic equation is derived to express this bearing function and test its design validation. A bearing is designed as an example to test the ability of the new technique, through the general characteristic equation, to offer a self-restriction bearing i.e. restrictor-less bearing. Ten figures (1-10) and two tables (12) express the calculations results.

\section{Discussion}

Because of the novelty, the discussion may contain some necessary details needed to reveal the author opinion and options.

\subsection{Bearing Design Technique}

The design technique is merely based on unifying the dominant bearing parameters, the supply pressure, the lubricant flow rate and seat inlet orifice diameter $\left(P_{s} . q, d_{i o}\right)$ through the derived general characteristic equation to ease and simplify the bearing selection (regardless of it is with or without restrictor) depending only on its configuration that succeeds to meet the required application demands.

\subsection{General Characteristic Equation}

This new equation is derived through relating the capillary and orifice restrictors of the bearing and equating the two ratios $\left(m_{c}\right.$ and $\left.m_{o}\right)$ to get equation (9). The equation expresses the characteristic of this type of bearing in general. Despite of its simplicity the equation relates the eccentricity (e), the lubricant flow rate, the central pressure ratio, central and supply pressures $\left(p_{i}, p_{s}, \beta\right)$; through $(Q)$; the bearing inlet orifice diameter and the restrictor diameter; through $(\mathrm{m})$. Hence, it could be truly considered as the General Characteristic Bearing Equation, it controls the bearing parameters and never act the bearing outside its domination.

Equating the ratio $(m)$ with unity makes the restrictor parameter $(m)$ disappear yielding equation (10). Now, this equation does not point to or contain a restrictor, which theoretically means that it expresses a restrictor-less bearing. The physical meaning of disappearing $(\mathrm{m})$ is that the inlet bearing orifice does not only act as a flow passage but also as an orifice restrictor. The previous studies handling the subject of designing untraditional bearings depend on the bearing outlet passage to act as a restrictor where the bearings are called as self compensation [5-10].

So, the author prefers to call the present bearing, 
mathematically, as a restrictor-less bearing or, physically as a self-restriction bearing.

For the aforementioned reasons the author chooses the self-restriction bearing as a name of this new bearing.

\subsection{The Eccentricity}

The odd figures (1-7 a) and Table 1 show the determination of the eccentricity values for the different baring configurations.

\subsection{Load Carrying Capacity}

Table 1 shows that the partial un-recessed bearing configuration has failed to carry the required load.

\subsection{The Temperature Rise and Distribution}

The temperature rise is theoretically and numerically calculated.

\subsubsection{The Theoretical Solution}

Equation (22) is applied to calculate the temperature rise but it is impotent to help the designer finding the feature of this rise.

\subsubsection{The Numerical Solution}

While Equation (22) is unable to give the designer an idea about the temperature rise feature equation (23) can do.

\subsubsection{The Calculation Results}

The odd figures (1-7 c) express the temperature variation along the lubricant passage which got through the numerical solution of equation (23). Figures $(5,7$ c) show that the temperature has gone up to $650 C$ which makes the hemispherical bearing configuration with or without recess unsuitable for the design required conditions in spite of its stiffness which will be discussed in the next item to assure its invalidity.

\subsection{The Bearing Stiffness $(\lambda)$}

The stiffness in the author previous studies hasn't been handled in details.

\subsubsection{The Numerical Solution}

This numerical solution depends on disturbing the bearing eccentricity (e) through changing it by an infinitesimal quantity resulting in changing the radial thickness $\left(h_{r}\right)$, the pressure $(p)$ and in turn the load capacity $(w)$ by infinitesimal quantities. Hence, the stiffness is calculated as the ratio between the infinitesimal changes of $(w)$ and $\left(h_{r}\right)$. Because the radial thickness varies depending on its position as a function of $(\theta)$ the stiffness $\left(\lambda_{r}\right)$ also will be local depending on the $\left(h_{r}\right)$ position. The mean value of these local stiffness points is the mean stiffness.

The thickness $\left(h_{r}\right)$ is analyzed in the axial $\left(h_{a}\right)$ and transverse $\left(h_{t}\right)$ directions where the stiffness is based on the infinitesimal changes in these directions to be $\left(\lambda_{a}\right)$ and $\left(\lambda_{t}\right)$ equation (25-27).

\subsubsection{The Theoretical Solution}

A simple mathematical method is used to calculate the stiffness mathematically. The integration of the load capacity is differentiated relatively to the thickness to avoid the complications in the integration process and then the complications of re-differentiating the integrated load equation. It is seen from equation (28) that the stiffness is directly proportional to the pressure (times $\cos \theta$ ) which means that this stiffness has the same pressure feature as an internal entity and will be created and never be zero whenever a bearing process. As the pressure changes with $(\theta)$ and multiplied by $(\cos \theta)$, the stiffness is also local in the $(\theta)$ direction.

The mean value of these local stiffness points is the mean stiffness. From the aforementioned clarification the author prefers to call this stiffness as the internal stiffness $\left(\lambda_{\theta}\right)$ and believes that this internal stiffness is responsible for the bearing resistance to the angular displacement and the self alignment property of this type of bearings.

\subsubsection{The Stiffness of the Designed Bearing}

Figures (odd 1-7 b \& d) show the pressure and the infinitesimal changes in the eccentricity and the load.

Figures (even 2-8) show the stiffness in the different directions, as illustrated before, of the four designed bearing configurations. Table 1 compares between these different configurations to select the best one meets the design application demands.

Figures $(6,8)$ show the invalidity of the hemispherical configuration with and without recess where the stiffness in the different directions are practically zero except the internal one which can never be zero as mentioned before.

\subsubsection{Selection of the Designed Bearing Configuration}

From figures (1-8) and Table 1 it is clear that the recessed partial hemispherical configuration is the only one succeeded to meet the design requirements.

The un-recessed partial hemispherical configuration failed to carry the required load while the other configurations failed because of their zero stiffness and their extremely high temperature rise.

\subsection{The Checking Results of the Selected Configuration}

Figures (9-10) and table 2 show the effect of the seat arc length $(\eta)$, the surface roughness $(\xi)$, the recess arc length $(y)$ and the viscosity variability $\left(K_{v}\right)$ on the design referring to the selected bearing configuration.

It could be seen that the increase in $(\eta)$ leads to increase the load and decrease the stiffness while the increase in $(\xi)$ leads to slight decrease and increase in the load and the stiffness respectively. Treating the lubricant as a constant viscosity fluid $\left(K_{v}=0\right)$ shows increase in the load and decrease in the stiffness. The only parameter which shows increase in both of the load and stiffness is (y) i.e. the increase in the recess angle $\left(\theta_{r}\right)$ leads to improve, to some extent, the bearing design depending on the design requirements. 
Table 2. Comparison between different parameters effects on the bearing design.

\begin{tabular}{llllll}
\hline Bearing & Ref. & $\eta$ & $\boldsymbol{\xi}$ & $\mathbf{y}$ & $\mathbf{K v}$ \\
parameters & bearing & $\mathbf{0 . 8 5}$ & $\mathbf{1 . 0}$ & $\mathbf{0 . 4}$ & $\mathbf{0 . 0}$ \\
\hline$\varphi \mathrm{i}($ deg $)$ & 0.8 & 0.8 & 0.8 & 0.8 & 0.8 \\
$\varphi \mathrm{r} \approx$ & 21.9 & 23 & 21.9 & 21.9 & 21.9 \\
$\varphi \mathrm{e} \approx$ & 72.9 & 76.5 & 72.9 & 72.9 & 72.9 \\
$\Delta(\mathrm{mm})$ & 5 & 5 & 5 & 5 & 5 \\
dio $\approx$ & 1.4 & 1.4 & 1.4 & 1.4 & 1.4 \\
$\theta \mathrm{i}($ degree $)$ & 0.8 & 0.8 & 0.8 & 0.8 & 0.8 \\
$\theta \mathrm{r} \approx$ & 21.8 & 22.9 & 21.8 & 29 & 21.8 \\
$\theta \mathrm{e} \approx$ & 72.7 & 76.29 & 72.7 & 72.7 & 72.7 \\
$\beta($ dim-less $)$ & $2 / 3$ & $2 / 3$ & $2 / 3$ & $2 / 3$ & $2 / 3$ \\
$\mathrm{Ps}(\mathrm{MN} / \mathrm{m} 2)$ & 2.41 & 2.41 & 2.41 & 2.41 & 2.41 \\
$\mathrm{e}(\mathrm{mm})$ & 0.1682 & 0.1873 & 0.164 & 0.136 & 0.174 \\
he $(\mu \mathrm{m})$ & 50 & 44.4 & 48 & 48.5 & 51.6 \\
$\mathrm{Ke}($ dim-less $)$ & $1 / 300$ & $1 / 267$ & $1 / 303$ & $1 / 306$ & $1 / 287$ \\
$\mathrm{~S} \approx$ & 1 & 1 & 1 & 1 & 1 \\
$\mathrm{w}(\mathrm{KN})$ & 20.718 & 24.788 & 20.38 & 22.9 & 22.2 \\
$\mathrm{fs}=(\mathrm{w} / \mathrm{L})$ & 1.0359 & 1.24 & 1.019 & 1.14 & 1.1 \\
$\mathrm{Q}($ dim-less $)$ & 0.6725 & 0.4852 & 0.707 & 0.73 & 0.394 \\
$\mathrm{q}(\mathrm{m} / \mathrm{hr})$ & 0.1429 & 0.1429 & 0.143 & 0.143 & 0.143 \\
$\mathrm{Pp}(\mathrm{N} . \mathrm{m} / \mathrm{s})$ & 95.77 & 95.43 & 94.7 & 94.6 & 61.8 \\
$\lambda \mathrm{A}(\mathrm{N} / \mu \mathrm{m})$ & 188.4 & 129.2 & 213.5 & 255 & 104 \\
$\lambda \mathrm{R} \approx$ & 110 & 66.2 & 124 & 148 & 61 \\
$\lambda \mathrm{T} \approx$ & 347 & 195 & 393 & 469 & 192 \\
$\lambda \theta \approx$ & 132 & 127 & 134 & 147 & 134 \\
$\Delta \mathrm{Tn}($ deg. c) & 2.86 & 2.92 & 2.9 & 2.8 & 2.87 \\
$\Delta \mathrm{Tth}($ deg. c) & 1.48 & 1.48 & 1.46 & 1.46 & 1 \\
\hline & & & & & \\
\hline
\end{tabular}

\section{Conclusion}

Based on an unconventional design technique a new characteristic equation is derived to give the designer the ability and flexibility to easily design conventional or unconventional bearings.

The equation proved its validity through offering an unconventional bearing, self restriction bearing, as a design example.

The bearing's inlet orifice diameter, the radius and the lubricant properties (viscosity and density) play the major role in determining the supply pressure in this new design technique.

The use of the maximum possible inertia effect enables the designer to minimize the supply pressure needed to lift the required load.

This design technique proved the inadequacy of the hemispherical bearing configuration with or without recess to meet the high inertia design requirement and the impotence to be a self restriction bearing due to the highly temperature rise and zero stiffness; Also the un-recessed partial hemispherical configuration failed to be a self restriction bearing due to its inability to lift the required load.

The only configuration that could be a self restriction bearing is the recessed partial hemispherical one. Its recess arc length plays the major role in improving its stiffness and increasing its ability to lift the loads.

The seat arc length $(\eta)$, the surface roughness $(\xi)$ and the viscosity variability $(K v)$ do not have the ability to improve the bearing design i.e. while increasing the load capacity the stiffness decreases and vice versa.

\section{Appendix}

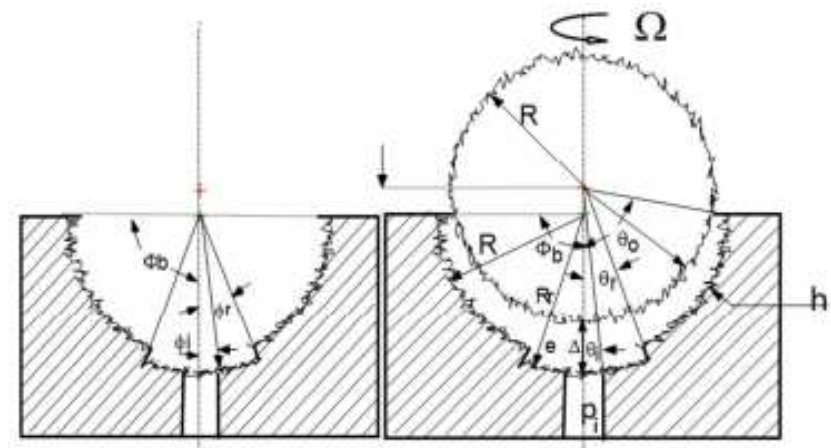

Figure A1. Bearing Configuration.

\section{Mathematical Expressions}

All mathematical equations related to this type of bearings could be found in Yacout [1-4] and the necessary ones which serve this design are listed.

Dimensionless speed parameter

$$
\begin{aligned}
& p_{i}=\left(\frac{2}{3}\right) p_{s} \\
& S=\frac{3 \rho \Omega^{2} R^{2}}{40 p_{i}}
\end{aligned}
$$

Pressure distribution $(P)$

$$
\begin{aligned}
& P=\frac{\alpha A}{b^{2}+1}\left\{\frac{1}{2 b^{2}} \ln \left(1+b^{2} \sec ^{2} \theta\right)+\ln (\tan (\theta)\} \ldots \ldots\right. \\
& -\frac{\alpha A k_{v}}{2 b^{2}}\left\{\ln \left(\frac{1+\sin \theta}{1-\sin \theta}\right)+\frac{1}{\sqrt{b^{2}+1}} \ln \left(\frac{\sqrt{b^{2}+1}-\sin \theta}{\sqrt{b^{2}+1}+\sin \theta}\right)\right\} \\
& -2 S \cos ^{2} \theta+D
\end{aligned}
$$

Where:

$D=B$ at the recess zone.

$D=B \& \alpha=1$ at the seat zone.

$$
\begin{gathered}
B=2 S \cos ^{2} \theta_{e}-A\left[\frac { 1 } { b ^ { 2 } + 1 } \left\{\frac{1}{2 b^{2}} \ln \left(1+b^{2} \sec ^{2} \theta_{e}\right)+\ln \left(\tan \left(\theta_{e}\right)\right\}\right.\right. \\
\left.-\frac{k_{v}}{2 b^{2}}\left\{\ln \left(\frac{1+\sin \theta_{e}}{1-\sin \theta_{e}}\right)+\frac{1}{\sqrt{b^{2}+1}} \ln \left(\frac{\sqrt{b^{2}+1}-\sin \theta_{e}}{\sqrt{b^{2}+1}+\sin \theta_{e}}\right)\right\}\right] \\
A=\frac{1+2 S\left(\cos ^{2} \theta_{i}-\cos ^{2} \theta_{e}\right)}{L_{1}-L_{2}} \\
L_{1}=\left[\frac{1}{b^{2}+1}\left\{\frac{1}{2 b^{2}} \ln \frac{\left(1+b^{2} \sec ^{2} \theta_{r}\right)}{\left(1+b^{2} \sec ^{2} \theta_{e}\right)}+\ln \frac{\left(\tan \left(\theta_{r}\right)\right.}{\left(\tan \left(\theta_{e}\right)\right.}\right\}\right. \\
-\frac{k_{v}}{2 b^{2}}\left\{\ln \left(\frac{1+\sin \theta_{r}}{1-\sin \theta_{r}} * \frac{1-\sin \theta_{e}}{1+\sin \theta_{e}}\right)+\right. \\
\left.\left.\frac{1}{\sqrt{b^{2}+1}} \ln \left(\frac{\sqrt{b^{2}+1}-\sin \theta_{r}}{\sqrt{b^{2}+1}} * \frac{\sqrt{b^{2}+1}+\sin \theta_{r}}{\sqrt{b^{2}+1}}-\sin \theta_{e}\right)\right\}\right]
\end{gathered}
$$




$$
\begin{aligned}
& L_{2}=\left[\frac{\alpha}{b^{2}+1}\left\{\frac{1}{2 b^{2}} \ln \frac{\left(1+b^{2} \sec ^{2} \theta_{r}\right)}{\left(1+b^{2} \sec ^{2} \theta_{i}\right)}+\ln \frac{\left(\tan \left(\theta_{r}\right)\right.}{\left(\tan \left(\theta_{i}\right)\right.}\right\}\right. \\
& -\frac{\alpha k_{v}}{2 b^{2}}\left\{\ln \left(\frac{1+\sin \theta_{r}}{1-\sin \theta_{r}} * \frac{1-\sin \theta_{i}}{1+\sin \theta_{i}}\right)+\right. \\
& \left.\left.\frac{1}{\sqrt{b^{2}+1}} \ln \left(\frac{\sqrt{b^{2}+1}-\sin \theta_{r}}{\sqrt{b^{2}+1}+\sin \theta_{r}} * \frac{\sqrt{b^{2}+1}+\sin \theta_{i}}{\sqrt{b^{2}+1}-\sin \theta_{i}}\right)\right\}\right]
\end{aligned}
$$

Flow rate

$$
\begin{aligned}
& Q=-A=\frac{6 \mu q}{\pi e^{3} \beta p_{s}} \\
& q=\frac{\pi e^{3} \beta p_{s} Q}{6 \mu}
\end{aligned}
$$

Dimensionless load carrying capacity $(W)$

$$
W=\sin ^{2} \theta+2\left[\left(a_{1}+b_{1}\right)-\left(b_{2}-a_{2}\right)\right]
$$

Where:

$$
\begin{gathered}
a_{1}=A\left[\frac{b^{2}+\cos ^{2} \theta}{4 b^{2}\left(b^{2}+1\right)} \ln \left(b^{2}+\cos ^{2} \theta\right)-\frac{\sin ^{2} \theta}{2\left(b^{2}+1\right)} \ln (\sin \theta)-\right. \\
\left.\frac{\cos ^{2} \theta}{2 b^{2}} \ln (\cos \theta)\right]_{\theta_{e}}^{\theta_{r}}+\frac{S}{2}\left[\cos ^{4} \theta\right]_{\theta_{r}}^{\theta_{e}}-\frac{B}{2}\left[\cos ^{2} \theta\right]_{\theta_{r}}^{\theta_{e}} \\
a_{2}=\frac{K_{v} A}{4 b^{2}}\left[\frac{\left(\cos ^{2} \theta\right)}{2} \ln \frac{(1-\sin \theta)}{(1+\sin \theta)}-\left(\frac{b^{2}+\cos ^{2} \theta}{2 \sqrt{b^{2}}+1}\right) \ln \frac{\left(\sqrt{b^{2}+1}-\sin \theta\right)}{\left(\sqrt{b^{2}+1}+\sin \theta\right)}\right]_{\theta_{r}}^{\theta_{e}} \\
\left.\frac{\cos ^{2} \theta}{2 b^{2}} \ln (\cos \theta)\right]_{\theta_{r}}^{\theta_{i}}+\frac{S}{2}\left[\cos ^{4} \theta\right]_{\theta_{i}}^{\theta_{r}}-\frac{B_{r}}{2}\left[\cos ^{2} \theta\right]_{\theta_{i}}^{\theta_{r}} \\
b_{2}^{2}\left(b^{2}+\frac{K_{v}^{2} A_{r}}{4 b^{2}}\left[\frac{\left(\cos ^{2} \theta\right)}{2} \ln \left(b^{2}+\cos ^{2} \theta\right)-\frac{(1-\sin \theta)}{(1+\sin \theta)}-\right.\right. \\
\left.\left(\frac{b^{2}+\cos ^{2} \theta}{2 \sqrt{b^{2}}+1}\right) \ln \frac{\left(\sqrt{b^{2}+1}-\sin \theta\right)}{\left(\sqrt{b^{2}+1}+\sin \theta\right)}\right]_{\theta_{i}}^{\theta_{r}} \\
A_{r}=\alpha A
\end{gathered}
$$

Dimensionless frictional torque (M)

$$
M=z_{b} M_{r}+M_{s}
$$

Where:

$$
\begin{aligned}
& M_{r}=\left[\frac{\cos ^{2} \theta}{2}+\left(\sigma^{2}-1\right) \ln (\cos \theta)+-\frac{\sigma^{2}}{2 \cos ^{2} \theta}\right]_{\theta_{i}}^{\theta_{r}}- \\
& K_{v}\left[\left(\sigma^{2}-1\right)\{\sin \theta-\ln (\tan \theta+\sec \theta)\}-\right. \\
& \left.\frac{\sigma^{2}}{2}\{\ln (\sec \theta+\tan \theta)-\sec \theta \tan \theta\}-\frac{\sin ^{3} \theta}{3}\right]_{\theta_{i}}^{\theta_{r}}
\end{aligned}
$$

$$
\begin{gathered}
M_{s}=\left[\frac{\cos ^{2} \theta}{2}+\left(\sigma^{2}-1\right) \ln (\cos \theta)+-\frac{\sigma^{2}}{2 \cos ^{2} \theta}\right]_{\theta_{r}}^{\theta_{e}}- \\
K_{v}\left[\left(\sigma^{2}-1\right)\{\sin \theta-\ln (\tan \theta+\sec \theta)\}-\right. \\
\left.\frac{\sigma^{2}}{2}\{\ln (\sec \theta+\tan \theta)-\sec \theta \tan \theta\}-\frac{\sin ^{3} \theta}{3}\right]_{\theta_{r}}^{\theta_{e}} \\
b_{2}=\frac{K_{v} A_{r}}{4 b^{2}}\left[\frac{\left(\cos ^{2} \theta\right)}{2} \ln \frac{(1-\sin \theta)}{(1+\sin \theta)}-\right. \\
\left.\left(\frac{b^{2}+\cos ^{2} \theta}{2 \sqrt{b^{2}}+1}\right) \ln \frac{\left(\sqrt{b^{2}+1}-\sin \theta\right)}{\left(\sqrt{b^{2}+1}+\sin \theta\right)}\right]_{\theta_{i}}^{\theta_{r}}
\end{gathered}
$$

Pump power

$$
\begin{aligned}
& p_{p}=q \cdot p_{s} \\
& P_{p}=\left(\frac{\pi \beta p_{s}^{2} e^{3}}{6 \mu}\right) Q
\end{aligned}
$$

Frictional power

$$
\begin{aligned}
& p_{f}=m \cdot \Omega \\
& P_{f}=\left(\frac{2 \pi \mu R^{4} \Omega^{2}}{e}\right) M \\
& P_{t}=P_{f}+P_{p}
\end{aligned}
$$

\section{Temperature rise and distribution}

The temperature rise could be determined theoretically as:

$$
\Delta T=\frac{P_{P}}{\rho q C_{v}}
$$

Or numerically as:

$$
\begin{aligned}
& \frac{d T}{d \theta}=\left(\frac{p_{i}}{\rho c_{v}}\right) \frac{\left(\frac{d P}{d \theta}\right)^{2}-4 S\left(\frac{d P}{d \theta}\right) \sin (2 \theta)+\frac{(S \sin 2 \theta)^{2}}{0.21}}{\left(2 \sin 2 \theta-\frac{d P}{d \theta}\right)} \\
& +\frac{(\text { Const }) \sin ^{2} \theta \sec ^{4} \theta\left(1-k_{v} \sin \theta\right)^{2}}{\left(2 \sin 2 \theta-\frac{d P}{d \theta}\right)}=X
\end{aligned}
$$

$$
\begin{aligned}
& \frac{\Delta T}{\Delta \theta}=X \\
& T_{n}=T_{n-1}+X \Delta \theta \\
& T_{1}=T_{i} \\
& \text { Const }=\left(\frac{160 S \mu_{i}^{2}}{\rho p_{i} R^{2} K_{e}^{4}}\right)
\end{aligned}
$$

The stiffness numerical solution

$$
\begin{aligned}
& \lambda=-d w / d h \\
& h=e \cos \theta
\end{aligned}
$$


Through an infinitesimal change in the eccentricity (e), an infinitesimal change in the load, in $(\theta)$ and in the film thickness (h) will dependably occur. So, the numerical solution could be as:

$$
\begin{aligned}
& w=a \beta P_{s} W \\
& \lambda=-\frac{d w}{d h}=-a P_{s}[\beta \bar{W}+W \bar{\beta}] \\
& \bar{W}=\frac{\Delta W}{\Delta h}, \quad \bar{\beta}=\frac{\Delta \beta}{\Delta h} \\
& \Delta W=W_{2}-W_{1} \quad, \quad \Delta \beta=\beta_{2}-\beta_{1} \\
& \Delta h=h_{2}-h_{1} \\
& \Delta \beta=\frac{6 \mu q}{\pi P_{s}}\left[\frac{1}{e_{2}^{3} Q_{2}}-\frac{1}{e_{1}^{3} Q_{1}}\right] \\
& h_{2}=e_{2} \cos (\theta)_{2} \\
& h_{1}=e_{1} \cos (\theta)_{1} \\
& (\theta)_{2}=\left(\theta_{i}: \theta_{e}\right)_{2} \\
& (\theta)_{1}=\left(\theta_{i}: \theta_{e}\right)_{1}
\end{aligned}
$$

From equations (24-26), the localized stiffness $(\lambda)_{l}$ could be calculated in turn the mean stiffness as:

$$
\lambda=\sum_{l=1}^{l}(\lambda)_{l}
$$

Internal bearing stiffness $\left(\lambda_{\theta}\right)$

Theoretically, the bearing stiffness could be calculated as:

$$
\begin{gathered}
\lambda=-\frac{d w}{d h} \\
h=e \cos \theta \\
w=\pi R^{2} p_{i} \sin ^{2} \theta_{i}+2 \pi R^{2} \int_{\theta_{i}}^{\theta_{o}} p \sin \theta \cos \theta d \theta \\
\lambda=-\frac{d w}{d h}=-\frac{d w}{e d(\cos \theta)}=\frac{d w}{e \sin \theta d \theta} \\
\frac{d w}{d \theta}=\frac{1}{d \theta}\left[\pi R^{2} p_{i} \sin ^{2} \theta_{i}+2 \pi R^{2} \int_{\theta_{i}}^{\theta_{o}} p \sin \theta \cos \theta d \theta\right] \\
\frac{d w}{d \theta}=2 \pi R^{2} p \sin \theta \cos \theta \\
\lambda=\left(\frac{1}{e \sin \theta}\right) x 2 \pi R^{2} p \sin \theta \cos \theta
\end{gathered}
$$

$$
\begin{aligned}
& \lambda=\left(\frac{2 \pi R^{2} p}{e}\right) \cos \theta \\
& \lambda_{\theta}=\sum_{\theta=\theta_{i}}^{\theta_{e}}(\lambda)_{\theta}
\end{aligned}
$$

\section{Nomenclature}

$A=-\left(6 \mu q / e^{3} \pi p_{i}\right)$
$A_{p w a}=$ Projected wet area

$a=$ Bearing projected area $\left(\pi R^{2}\right)$.

$C=-\left(6 \mu_{i} q / \pi\right)$.

$c_{v}=$ Lubricant specific heat $1880(\mathrm{~J} / \mathrm{Kg} . \mathrm{c})$

$E(f)=$ Expected value of.

$e=$ Eccentricity \& $e_{r}=\Delta+e$

$f=$ Dimensionless friction factor.

$F=$ Friction factor.

$H=$ Dimensionless film thickness.

$h=$ Film thickness $(e \cos \theta)$.

$h_{f}=$ Power facto.

$J_{\mathrm{f}}=$ Geometry factor $(1 / W)$.

$K_{\mathrm{v}}=$ Constant of viscosity variation

$K_{\mathrm{e}}=(e / R) \& K_{\mathrm{r}}=\left(e_{r} / R\right)$.

$\mathrm{L}=$ Load required to be lifted.

$m=$ Frictional torque.

$M=$ Dimensionless frictional torque $\left(m e / 2 \pi \mu \Omega R^{4}\right)$

$m_{c}=$ Capillary tube diameter ratio.

$m_{o}=$ Orifice diameter ratio.

$N=$ Shaft speed (r. p. s).

$P=$ Dimensionless pressure $\left(p / p_{i}\right)$

$p=$ pressure along the fluid film

$p_{i}=$ Inlet pressure.

$p_{s}=$ Supply pressure.

$Q=$ Dimensionless volume flow rate $(Q=-A)$.

$q=$ Volume flow rate.

$R=$ Bearing radius.

$R_{e}=$ Reynolds number.

$S=$ Speed parameter $\left(3 \rho \Omega^{2} R^{2} / 40 p_{i}\right)$.

$\mathrm{T}=$ Temperature

$W=$ Dimensionless load carrying capacity $\left(w / \pi R^{2} p_{i}\right)$

$w=$ Load carrying capacity.

$y=\frac{\phi_{r}}{\phi_{e}}$

$z_{b}=\left(e / e_{r}\right) \& \alpha=\left(z_{b}^{3}\right)$

$\beta=\left(p_{i} / p_{s}\right)$.

$\theta=$ Angle co-ordinate.

$\varphi_{e}=$ Seat outer rim angle.

$\varphi_{r}=$ Recess angle 


\author{
$\theta_{i}=$ Inlet flow angle. \\ $\theta_{\mathrm{e}}=$ Outlet flow angle. \\ $\Delta=$ Recess depth \\ $\Delta T=$ Temperature rise \\ $\rho=$ Lubricant density. \\ $\sigma=$ Dimensionless surface roughness parameter. \\ $\sigma_{o}^{2}=$ Variance of the film thickness. \\ $\lambda=$ Bearing stiffness. \\ $\lambda_{a}=$ Axial stiffness \\ $\lambda_{t}=$ Transversal stiffness \\ $\lambda_{r}=$ Radial stiffness \\ $\lambda_{\theta}=$ Internal stiffness \\ $\mu=$ Lubricant viscosity. \\ $\Omega=$ Rotational speed \\ Suffix

$\begin{array}{ll}\text { c } & \text { capillary } \\ \text { o } & \text { orifice } \\ \text { e } & \text { exit } \\ \text { i } & \text { inlet } \\ \text { th } & \text { theoretical } \\ \text { n } & \text { numerical }\end{array}$

\section{References}

[1] Ahmad W. Yacout, Ashraf S. Ismaeel, Sadek Z. assab, "The combined effects of the centripetal inertia and the surface roughness on the hydrostatic thrust spherical bearing performance", Tribolgy International Journal 2007, Vol. 40, No. 3, 522-532.

[2] Ahmad W. Y. Elescandarany, "The Effect of the fluid film variable viscosity on the hydrostatic thrust spherical bearing performance in the presence of centripetal inertia and surface roughness (Part 1 Un-recessed fitted bearing)", The International Journal of Mechanical Engineering and Applications 2018, Vol. 6, No. 1, pp. 1-12.

[3] Ahmad W. Y. Elescandarany, "The Effect of the fluid film variable viscosity on the hydrostatic thrust spherical bearing performance in the presence of centripetal inertia and surface roughness (Part2 Recessed fitted bearing)", The International Journal of Mechanical Engineering and Applications 2018, Vol. 6, No. 3, pp. 73-90.

[4] Ahmad Waguih Yacout Elescandarany, "Design of the
Hydrostatic Thrust Spherical Bearing with Restrictors (Fitted Type)“, The International Journal of Mechanical Engineering and Applications 2019 Vol. 7, No. 2, pp. 34-45.

[5] Kane N. R., Sihler J. and Slocum A. H. "A hydrostatic rotary bearing with angled surface self-compensation", Precision Engineering 2003, 5321, pp: 1-15.

[6] Xiaobo Z., Shengyi L., Ziqiang Y. and Jianmin W. "Design and Parameter Study of a Self-Compensating Hydrostatic Rotary Bearing" International Journal of Rotating Machinery, Volume 2013, Article ID 638193, pp: 1-10.

[7] Yuan K., De-Xing P., Yu-Hong H. and Sheng-Yan H." Design for static stiffness of hydrostatic bearings: double-action variable compensation of membrane-type restrictors and selfcompensation". Industrial Lubrication and Tribology 2014, Vol. 66, · No. 2, pp: 322-3343.

[8] Xu C. and Jiang S., "Analysis of the static characteristics of a self-compensation hydrostatic spherical hinge". J. Tribolohy T. ASME 2015; Vol. 137, No. 4: 044503-044503-5.

[9] Khaksea P. G., Phalleb V. M. and Manthac S. S. "Comparative Performance of a Non-recessed Holeentry Hybrid/Hydrostatic Conical Journal Bearing Compensated with Capillary and Orifice Restrictors", Journal of Tribology in Industry 2016, Vol. 38, No. 2, pp: 133-148.

[10] Zhifeng Li., Yumo W., Ligang C., Yongsheng Z., Qiang C. and Xiangmin D., "A review of hydrostatic bearing system: Researches and Applications", Advances in Mechanical Engineering 2017, Vol. 9, No. 10, pp: 1-28.

[11] Alexander Slocum, "Externally Pressurized Fluid Film Bearings", Precision Machine Design, ME EN 7960 - NonContact Bearings - Topic 11, pp: 1-47.

[12] Alexander Slocum, Paul Scagnetti, Nathan Kane and Christoph Brunner," Design of self-compensated, waterhydrostatic bearings" Journal of Precision Engineering 1995, Vol. 17, No. 3, pp: 173-185.

[13] Zhao Yang Dong, Sheng-Yen Hu, Chao-Ping Huang and Yuan Kang" Static Characteristic of Self-compensated Hydrostatic Bearing" Proceedings of the International Conference on Environmental Science and Sustainable Energy, 2017.

[14] Mohit Agarwal, "Non-Dimensional Parameters of a Membrane-Type Restrictor in an Opposed Pad Hydrostatic Bearing for High Static Stiffness", International Journal of Science and Research 2018, Vol. 7 No. 7, pp: 1306-1313.

[15] Antony Raymond Wong," Design of low cost hydrostatic bearing", Master thesis, Massachusetts Institute of Technology, 2019. 